\title{
Design of Novel GPR6 Inverse Agonists Using a Fragment Replacement Scaffold Hopping Approach ${ }^{\dagger}$
}

\author{
Israa Isawi 1,*, Paula Morales ${ }^{2}$, Dow Hurst ${ }^{1}$, Nadine Jagerovic ${ }^{2}$ and Patricia Reggio ${ }^{1}$ \\ 1 Chemistry \& Biochemistry Dept., UNCG, Greensboro, NC 27412, USA \\ 2 Instituto de Química Médica, Consejo Superior de Investigaciones Científicas, E-28006 Madrid, Spain \\ * Correspondence: ihisawi@uncg.edu; Tel.: +1-336-491-0330 \\ + Presented at the 2nd Molecules Medicinal Chemistry Symposium (MMCS): Facing Novel Challenges in \\ Drug Discovery, Barcelona, Spain, 15-17 May 2019.
}

Published: 7 August 2019

\begin{abstract}
The orphan G protein coupled receptor 6 (GPR6) is a cannabinoid-related Class A GPCR. It is highly expressed in the central nervous system and exhibits high constitutive activation of adenylyl cyclase. Several research groups have demonstrated that GPR6 represents a possible target for the treatment of neurodegenerative disorders such as Parkinson's, Alzheimer's, and Huntington's diseases. Several patents claim the use of a wide range of pyrazine derivatives as GPR6 inverse agonists for the treatment of Parkinson's disease and other dyskinesia syndromes. Using cyclic AMP accumulation assays in $h \mathrm{GPR} 6-\mathrm{CHO}$ cells as a readout, the most potent GPR6 pyridopyrazine inverse agonist compounds identified thus far have been found to display $\mathrm{IC}_{50}$ values in the low nanomolar range. A subset of these compounds was used here as starting points for the design of novel potent GPR6 inverse agonists using a core hopping approach. In parallel with the core hopping studies, we employed a recently constructed homology model of the GPR6 inactive state. The X-ray crystal structure of the Sphingosine-1-phosphate receptor 1 (S1P1) receptor structure was used as the template and the conformational memories technique was used to explore the conformational consequences of sequence differences between S1P1 and GPR6. The most potent GPR6 pyridopyrazine inverse agonists were docked in the resultant GPR6 inactive state model, first as tests of the model. Once we had identified the binding site of each inverse agonist, we used this site to identify amino acid sites in proximity that we can use to build additional interactions for ligands output from core hopping studies above. ADME properties and the absence of PAINS will also be considered for the hit selection process. These potential GPR6 chemotypes may serve as research tools for further understanding the biological role of this orphan receptor.
\end{abstract}

Keywords: GPR6; Scaffold Hopping; Parkinson's Disease

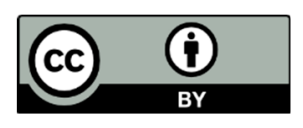

(c) 2019 by the authors. Licensee MDPI, Basel, Switzerland. This article is an open access article distributed under the terms and conditions of the Creative Commons Attribution (CC BY) license (http://creativecommons.org/licenses/by/4.0/). 